\title{
COMPARATIVE ANALYSIS MIRAS/PPN
}

D. BARTHES, M.O. MENNESSIER and F. GLEIZES

GRAAL, CNRS and Univ. Montpellier II, F 34095 Montpellier Cedex 5, France

and

A. LÈBRE

DASGAL, Obs. Paris-Meudon, F 92195 Meudon Principal Cedex, France

The spectrum of R Sct, a star firstly classified as an RV Tau one and located in the highest part of the instability strip, indicates a $\mathrm{Na}$ ejected shell at about $1000 \mathrm{R}_{*}$ with an expansion velocity close to $50 \mathrm{~km} / \mathrm{s}$ (Lèbre and Gillet, 1991). This confirms the post AGB character of this star as already mentionned by Jura (1986). So it is interesting to compare the R Sct with some "classical" RV Tauri (AC Her and U Mon) and some "exceptional" miras (R Cen, U CMi and R Nor) with light curves exhibiting a double maximum and with spectral types earlier than those of the other miras with similar periods.

Such a comparison using the power spectra of their light curves gives the following results:

- $\quad$ R Sct and the three peculiar miras are pulsating on the fundamental mode with resonance with the first overtone, instead of the classical miras. This is indicated by the agreement between the power spectra and the linear nonadiabatic models of Tuchman et al.(1992)

$\left(0.5<\mathrm{M}_{*} / \mathrm{M}_{\odot}<0.8 ; 10^{3}<\mathrm{L}_{*} / \mathrm{L}_{\odot}<10^{4}\right)$.

- The RV Tau stars AC Her and U Mon are pulsating on the first overtone with resonance with the fundamental mode.

This is a possible indication that $\mathrm{R}$ Cen, $\mathrm{R}$ Nor and $\mathrm{U}$ CMi are leaving the Mira stage after a mode switching from the first overtone to the fundamental mode. $\mathrm{R}$ Sct is confirmed as a post-AGB star and probably a PPN.

\section{References}

Jura M., 1986, Ap.J., 309 ,732

Lèbre A., Gillet D., 1991 A\&A, 251, 549

Tuchman Y., Lèbre A., Mennessier M.O., Yarri A., A\&A, to be submitted 